\title{
Sun Exposure: Beyond the Risks
}

\author{
Catarina Soares Queirós ${ }^{1}$, João Pedro Freitas, $\mathrm{MD}^{1}$
}

1 Serviço de Dermatologia do Hospital de Santa Maria, Centro Hospitalar e Universitário de Lisboa Norte, Lisbon, Portugal

Key words: sun, vitamin D, skin neoplasms, nitric oxide

Citation: Queirós CS, Freitas JP. Sun exposure: beyond the risks. Dermatol Pract Concept. 2019;9(4):249-252. DOI: https://doi. org/10.5826/dpc.0904a01

Accepted: July 23, 2019; Published: October 31, 2019

Copyright: @2019 Soares Queirós et al. This is an open-access article distributed under the terms of the Creative Commons Attribution License, which permits unrestricted use, distribution, and reproduction in any medium, provided the original author and source are credited.

Funding: None.

Competing interests: The authors have no conflicts of interest to disclose.

Authorship: All authors have contributed significantly to this publication.

Corresponding author: Catarina Soares Queirós, MD, Serviço de Dermatologia do Hospital de Santa Maria, Centro Hospitalar e Universitário de Lisboa Norte, Avenida Prof. Egas Moniz, 1649-035, Lisboa, Portugal. Email: catarina.squeiros@gmail.com

ABSTRACT Excessive solar exposure presents well-recognized risks and side effects, solar radiation being the most important environmental factor concerning skin cancer. In the last few years, several connections between solar exposure and prevention and/or treatment of several diseases have been discussed, with studies suggesting that regular solar exposure may be beneficial for conditions such as colorectal, breast, prostate, and pancreatic cancer; non-Hodgkin lymphoma; arterial hypertension; obesity; type 2 diabetes mellitus and metabolic syndrome; nonalcoholic hepatic steatosis; multiple sclerosis; Alzheimer disease; and several psychiatric disturbances. In most cases, UV radiation's beneficial effects are mediated through vitamin $\mathrm{D}$; however, studies show that in other instances other mediators are responsible for these associations, specifically nitric oxide. Moderation is therefore essential, as a strict strategy of total sun avoidance may be inadequate.

\section{Introduction}

Sunlight is composed of a spectrum of radiations that span from infrared to visible and UV light; UV radiation itself is further subdivided into UVA, UVB, and UVC. UVC radiation is blocked by stratosphere gases, and therefore only UVA and UVB rays reach the earth's surface [1].

Excessive solar exposure presents well-recognized risks and side effects, solar radiation being the most important environmental risk factor for skin cancer, the main cause of photoaging, and also an exacerbating factor for several photo-aggravated dermatoses [2,3]. However, in the last few years a variety of connections between solar exposure and prevention and/or treatment of several diseases have been discussed, with studies suggesting that regular solar exposure may have more benefits than previously thought [4]. The big question is, therefore, to which point actual practices of sun avoidance bring effective benefits to our health.

\section{Mechanisms of Action of Ultraviolet Radiation}

In the past, benefits of sun exposure were attributed only to vitamin D. Effectively, the most well-known advantage of sun exposure is the synthesis of vitamin $\mathrm{D}$, which is important for several physiological functions, UVB radiation being the most responsible for vitamin $\mathrm{D}$ levels in the vast majority of the population [5]. Vitamin D production starts with the 
reaction of 7-deoxycholesterol, produced in abundant quantity in the skin, with UVB radiation. This reaction leads to the production of cholecalciferol, which is transported to the liver, where it is hydroxylated at position 25 (a step catalyzed by the microsomal enzyme 25 -hydroxylase), thus producing calcifediol. Calcifediol is then released into the circulation, reaching the kidneys, where it is again hydroxylated at position 1- $\alpha$ (a step catalyzed by the enzyme hydroxyvitamin $D_{3}$ 1-a-hydroxylase), leading to the formation of calcitriol, the most active form of vitamin $\mathrm{D}$ and the one responsible for most of its effects [5].

Vitamin D is important when it comes to the absorption of calcium originated in the diet, therefore being essential to the maintenance of an adequate bone mineral density, its deficiency being associated with an increased risk of osteoporosis and rickets [6]. Apart from these effects, recent studies also suggest that vitamin D deficit possibly correlates with disease activity in rheumatoid arthritis [7] and that an increase in vitamin D levels in osteoarthritis may be associated with an improvement in life quality and muscular strength in these patients [8].

Recently, other mechanisms have been proposed to explain the beneficial association between solar exposure and certain diseases [4]; these include the action of nitric oxide, immunomodulation, the production of melatonin and serotonin, and the effect of light in circadian rhythms [2]. The role of nitric oxide is one of the most studied mechanisms; UVA radiation induces the release of this molecule from its storages in the skin, as well as its translocation to the blood. Here, nitric oxide is responsible for a variety of effects such as vasodilation and decrease of peripheral vascular resistance with a consequent decrease of blood pressure and suppression of glucose intolerance and insulin resistance [9]. As far as immunomodulation is concerned, it is known that both UVA and UVB have a direct immunosuppressor effect, by induction of cytokines such as tumor necrosis factor- $\alpha$ (TNF- $\alpha$ ) and interleukin-10 (IL-10) and stimulation of T regulatory cells. This mechanism may explain the benefit of sun exposure in the protection from certain autoimmune disorders such as multiple sclerosis or rheumatoid arthritis [10]. Other less well-studied mechanisms include the synthesis of endorphins and photodegradation of folic acid [2].

\section{Benefits of Sun Exposure}

The most-recognized benefits of sun exposure include the prevention and treatment of several dermatoses (such as psoriasis and eczema), vitamin D synthesis (important to normal bone and muscle physiology), and prevention and treatment of seasonal affective disorder. All these links are well established and supported, UV radiation being used in the treatment of some of these conditions, such as psoriasis.
Nevertheless, in recent years, several previously unrecognized connections between solar exposure and a decreased risk of some diseases have been proposed. Some studies have even demonstrated a reduction in all-cause mortality or cardiovascular mortality in patients with regular and moderate sun exposure [10].

\section{Cancer}

Cancer prevention is one of the areas of greatest interest concerning the benefits of vitamin D and, therefore, of sun exposure. Indeed, in the past decade numerous studies supported a strong association between UV exposure and a decreased risk of several cancers, namely breast, colon, ovary, and prostate cancer and non-Hodgkin lymphoma [5].

Recently, in a systematic review from van der Rhee et al on this topic, it was concluded that chronic regular solar exposure (as opposed to intermittent exposure) seems to have a protective effect against the incidence of colorectal, breast, and prostate cancer and non-Hodgkin lymphoma [11]. Focusing on breast cancer, in the following year a meta-analysis from Mohr et al demonstrated a significant mortality reduction in patients with normal levels of vitamin $\mathrm{D}$, when compared with patients with insufficient levels of this vitamin [12], thus confirming the previously described inverse link between solar exposure and breast cancer.

Although vitamin D has a strong influence on the effect of solar exposure on cancer prevention, it is not the only explanation for this association, which is not entirely reproduced by nutritional supplementation [1]. Therefore, other proposed mechanisms include immunomodulation induced by sun exposure and the effect of melatonin on the regulation of circadian rhythms, whose disruption is associated with carcinogenesis [2].

\section{Cardiovascular Diseases}

\section{Arterial Hypertension}

Several recent studies have evaluated the effects of UV exposure on blood pressure, and a protector effect seems to exist [13]. This effect does not seem to be dependent on its influence on vitamin D levels, but on the release of nitric oxide from skin storages upon solar exposure, with consequent vasodilation, as previously explained [9].

\section{Type 2 Diabetes Mellitus and Obesity}

In 2017, a study from Gorman et al on the effect of solar exposure in the development of obesity and type 2 diabetes mellitus concluded that continuous or regular sun exposure may control the appearance of both conditions [1]. In fact, in the last few years several studies have demonstrated the existence of an inverse correlation between body mass index and serum levels of vitamin $\mathrm{D}$, although in these observa- 
tional studies it is difficult to establish a cause-effect relation between these 2 variables [14]. On the other hand, the benefits of sun exposure in these settings may result through other mediators, namely through the release of nitric oxide from the skin after sun exposure or via secretion of a-melanocyte-stimulating hormone ( $\alpha-\mathrm{MSH})$ [1].

After regular and moderate sun exposure, patients with type 2 diabetes mellitus show an increase in insulin secretion and a decrease in high-sensitivity $\mathrm{C}$ reactive protein (an inflammatory marker). Regarding the high prevalence and mortality of this condition, this is an area where further studies are crucial to clarify these potential associations [1].

\section{Metabolic Syndrome}

Taking into account the proposed benefits of UV radiation exposure on arterial hypertension, obesity, and type 2 diabetes mellitus, it is easy to recognize that solar exposure may also bring benefits to patients with metabolic syndrome. Indeed, a study from Vitezova et al showed that higher levels of vitamin D are associated with a lower prevalence of metabolic syndrome in middle-aged and older people, even after adjusting for body mass index [15].

\section{Neurological Diseases}

\section{Multiple Sclerosis}

As far as multiple sclerosis is concerned, several studies demonstrated an increased risk of developing this disease in individuals with insufficient solar exposure, probably due to mechanisms unrelated to vitamin D $[11,13]$. In addition, prospective and case-control studies in Caucasian patients suffering from multiple sclerosis revealed a decrease in mortality risk as sun exposure hours increased [4].

\section{Dementia}

Recent studies have pointed toward the existence of a close relationship between low levels of vitamin D and the development of cognitive deficit and dementia. In particular, a prospective study from Littlejohns et al revealed that patients with vitamin D deficit have a 2 -fold increased risk of developing Alzheimer disease compared with individuals with normal levels of this same vitamin [16]. Although more evidence is needed, these findings may well be important in the future, as we are dealing with an increasingly elderly population, who therefore have a greater predisposition toward this type of pathology.

\section{Other Diseases}

\section{Psychiatric Disease}

One of the first recognized associations between solar exposure and development or treatment of certain diseases was in the field of psychiatry. Here, emphasis is given to the effect of solar exposure on the prevention and treatment of seasonal affective disorder, a benefit that seems to extend to other depressive states [2,17]. In this area, vitamin D seems to be one of the major mediators of the benefits of UV radiation [17].

\section{Hepatic Disease}

In the last few years, evidence has emerged in favor of a protective role of UV exposure on the development of nonalcoholic hepatic steatosis. This protective effect seems to be partially dependent on vitamin $\mathrm{D}$, but other factors are probably implicated [13].

\section{Conclusions}

In the last decade, several studies have proposed a strong connection between sun exposure and a decrease in the risk of developing certain diseases, including colorectal, breast, prostate, and pancreatic cancer; non-Hodgkin lymphoma; arterial hypertension; obesity; type 2 diabetes mellitus and metabolic syndrome; nonalcoholic hepatic steatosis; multiple sclerosis; Alzheimer disease; and several psychiatric disturbances. These potential relations are even more important if we consider that many of these conditions have a mortality rate that is higher than that of skin cancer. Frequently, beneficial effects of UV radiation are mediated through vitamin $\mathrm{D}$; however, in other circumstances, other mediators are responsible for these associations, more precisely nitric oxide. Therefore, it is easy to acknowledge that vitamin $\mathrm{D}$ supplementation does not replace adequate sun exposure.

Obviously, the other side of sun exposure must not be forgotten, as it is the major risk factor for skin cancer. Therefore, combinations of sun protection practices including the use of beach umbrellas, sunscreens with high sun protection factors, and protective clothing remain important and should be emphasized by dermatologists [18]. A strict strategy of total sun avoidance seems to be inadequate, so as with everything, moderation is essential.

\section{References}

1. Gorman S, Lucas RM, Allen-Hall A, Fleurya N, Feelischc M. Ultraviolet radiation, vitamin $\mathrm{D}$ and the development of obesity, metabolic syndrome and type-2 diabetes. Photochem Photobiol Sci. 2017;16(3):362-373.

2. van der Rhee HJ, de Vries E, Coebergh JW. Regular sun exposure benefits health. Med Hypotheses. 2016;97:34-37.

3. Weller RB. The health benefits of UV radiation exposure through vitamin D production or non-vitamin D pathways: blood pressure and cardiovascular disease. Photochem Photobiol Sci. 2017;16(3):374-380.

4. van der Rhee HJ, de Vries E, Coomans C, van de Velde P, Coebergh JW. Sunlight: for better or for worse? A review of positive and negative effects of sun exposure. Cancer Research Frontiers. 
2016;2(2):156-183. Available at: http://cancer-research-frontiers. org/wp-content/uploads/2016/04/CRF-2016-2-156.pdf.

5. Grant WB. Health benefits of solar UV-B radiation through the production of vitamin D: comment and response. Photochem Photobiol Sci. 2003;2(12):1307-1308; discussion 1308-1310.

6. Lips P, van Schoor NM. The effect of vitamin D on bone and osteoporosis. Best Pract Res Clin Endocrinol Metab. 2011;25(4):585591.

7. Caraba A, Crisan V, Romosan I, Mozos I, Murariu M. Vitamin D status, disease activity, and endothelial dysfunction in early rheumatoid arthritis patients. Dis Markers. 2017;2017:5241012.

8. Manoy P, Yuktanandana P, Tanavalee A, et al. Vitamin D supplementation improves quality of life and physical performance in osteoarthritis patients. Nutrients. 2017;9(8):799.

9. Liu D, Fernandez BO, Hamilton A, et al. UVA irradiation of human skin vasodilates arterial vasculature and lowers blood pressure independently of nitric oxide synthase. J Invest Dermatol. 2014;134(7):1839-1846.

10. Wright F, Weller RB. Risks and benefits of UV radiation in older people: more of a friend than a foe? Maturitas. 2015;81(4):425431.

11. van der Rhee HJ, Coebergh JW, de Vries E. Is prevention of cancer by sun exposure more than just the effect of vitamin D?
A systematic review of epidemiological studies. Eur J Cancer. 2013;49(6):1422-1436.

12. Mohr SB, Gorham ED, Kim J, Hofflich H, Garland CF. Meta-analysis of vitamin D sufficiency for improving survival of patients with breast cancer. Anticancer Res. 2014;34(3):1163-1166.

13. Hoel DG, Berwick M, de Gruijl FR, Holick MF. The risks and benefits of sun exposure 2016. Dermatoendocrinol. 2016;8(1):e1248325.

14. Earthman CP, Beckman LM, Masodkar K, Sibley SD. The link between obesity and low circulating 25 -hydroxyvitamin D concentrations: considerations and implications. Int J Obes (Lond). 2012;36(3):387-396.

15. Vitezova A, Zillikens MC, van Herpt TT, et al. Vitamin D status and metabolic syndrome in the elderly: the Rotterdam Study. Eur J Endocrinol. 2015;172(3):327-335.

16. Littlejohns TJ, Henley WE, Lang IA, et al. Vitamin D and the risk of dementia and Alzheimer disease. Neurology. 2014;83(10):920928.

17. Melrose S. Seasonal affective disorder: an overview of assessment and treatment approaches. Depress Res Treat. 2015;2015:178564.

18. Ou-Yang H, Jiang LI, Meyer K, et al. Sun protection by beach umbrella vs sunscreen with a high sun protection factor: a randomized clinical trial. JAMA Dermatol. 2017;153(3):304-308. 